\title{
Common fixed points of $R$-weakly commuting maps in generalized metric spaces
}

Mujahid Abbas', Safeer Hussain Khan ${ }^{2^{*}}$ and Talat Nazir ${ }^{1}$

\author{
* Correspondence: safeer@qu.edu. \\ qa \\ ${ }^{2}$ Department of Mathematics, \\ Statistics and Physics, Qatar \\ University, Doha 2713, Qatar \\ Full list of author information is \\ available at the end of the article
}

\begin{abstract}
In this paper, using the setting of a generalized metric space, a unique common fixed point of four $R$-weakly commuting maps satisfying a generalized contractive condition is obtained. We also present example in support of our result. 2000 MSC: 54H25; 47H10; 54E50.

Keywords: $R$-weakly commuting maps, compatible maps, common fixed point, generalized metric space
\end{abstract}

\section{Introduction and preliminaries}

The study of unique common fixed points of mappings satisfying certain contractive conditions has been at the center of rigorous research activity. Mustafa and Sims [1] generalized the concept of a metric, in which the real number is assigned to every triplet of an arbitrary set. Based on the notion of generalized metric spaces, Mustafa et al. [2-6] obtained some fixed point theorems for mappings satisfying different contractive conditions. Study of common fixed point theorems in generalized metric spaces was initiated by Abbas and Rhoades [7]. Abbas et al. [8] obtained some periodic point results in generalized metric spaces. While, Chugh et al. [9] obtained some fixed point results for maps satisfying property $p$ in G-metric spaces. Saadati et al. [10] studied some fixed point results for contractive mappings in partially ordered G-metric spaces. Recently, Shatanawi [11] obtained fixed points of $\Phi$-maps in G-metric spaces. Abbas et al. [12] gave some new results of coupled common fixed point results in two generalized metric spaces (see also [13]).

The aim of this paper is to initiate the study of unique common fixed point of four $R$-weakly commuting maps satisfying a generalized contractive condition in G-metric spaces.

Consistent with Mustafa and Sims [2], the following definitions and results will be needed in the sequel.

Definition 1.1. Let $X$ be a nonempty set. Suppose that a mapping $G$ :

$X \times X \times X \rightarrow R^{+}$satisfies:

$\mathbf{G}_{1}: G(x, y, z)=0$ if $x=y=z$;

$\mathbf{G}_{2}: 0<G(x, y, z)$ for all $x, y, z \in X$, with $x \neq y$;

$\mathbf{G}_{3}: G(x, x, y) \leq G(x, y, z)$ for all $x, y, z \in X$, with $y \neq z$;

$\mathbf{G}_{4}: G(x, y, z)=G(x, z, y)=G(y, z, x)=\cdots$ (symmetry in all three variables); and

$\mathbf{G}_{5}: G(x, y, z) \leq G(x, a, a)+G(a, y, z)$ for all $x, y, z, a \in X$.

Then $G$ is called a $G$-metric on $X$ and $(X, G)$ is called a $G$-metric space. 
Definition 1.2. A sequence $\left\{x_{n}\right\}$ in a $G$-metric space $X$ is:

(i) a G-Cauchy sequence if, for any $\varepsilon>0$, there is an $n_{0} \in N$ (the set of natural numbers) such that for all $n, m, l \geq n_{0}, G\left(x_{n}, x_{m}, x_{l}\right)<\varepsilon$,

(ii) a G-convergent sequence if, for any $\varepsilon>0$, there is an $x \in X$ and an $n_{0} \in N$, such that for all $n, m \geq n_{0}, G\left(x, x_{n}, x_{m}\right)<\varepsilon$.

A $G$-metric space on $X$ is said to be $G$-complete if every $G$-Cauchy sequence in $X$ is $G$-convergent in $X$. It is known that $\rightarrow 0$ as $n, m \rightarrow \infty$.

Proposition 1.3. Let $X$ be a G-metric space. Then the following are equivalent:

(1) $\left\{x_{n}\right\}$ is G-convergent to $x$.

(2) $G\left(x_{n}, x_{m}, x\right) \rightarrow 0$ as $n, m \rightarrow \infty$.

(3) $G\left(x_{n}, x_{n}, x\right) \rightarrow 0$ as $n \rightarrow \infty$.

(4) $G\left(x_{n}, x, x\right) \rightarrow 0$ as $n \rightarrow \infty$.

Definition 1.4. A $G$-metric on $X$ is said to be symmetric if $G(x, y, y)=G(y, x, x)$ for all $x, y \in X$.

Proposition 1.5. Every G-metric on $X$ will define a metric $d_{G}$ on $X$ by

$$
d_{G}(x, y)=G(x, y, y)+G(y, x, x), \quad \forall x, y \in X .
$$

For a symmetric $G$-metric,

$$
d_{G}(x, y)=2 G(x, y, y), \quad \forall x, y \in X .
$$

However, if $G$ is non-symmetric, then the following inequality holds:

$$
\frac{3}{2} G(x, y, y) \leq d_{G}(x, y) \leq 3 G(x, y, y), \quad \forall x, y \in X .
$$

It is also obvious that

$$
G(x, x, y) \leq 2 G(x, y, y) .
$$

Now, we give an example of a non-symmetric $G$-metric.

Example 1.6. Let $X=\{1,2\}$ and a mapping $G: X \times X \times X \rightarrow R^{+}$be defined as

$$
\begin{array}{cc}
(x, y, z) & G(x, y, z) \\
(1,1,1),(2,2,2) & 0 \\
(1,1,2),(1,2,1),(2,1,1) & 0.5 \\
(1,2,2),(2,1,2),(2,2,1) & 1 .
\end{array}
$$

Note that $G$ satisfies all the axioms of a generalized metric but $G(x, x, y) \neq G(x, y, y)$ for distinct $x, y$ in $X$. Therefore, $G$ is a non-symmetric $G$-metric on $X$.

In 1999, Pant [14] introduced the concept of weakly commuting maps in metric spaces. We shall study $R$-weakly commuting and compatible mappings in the frame work of $G$-metric spaces.

Definition 1.7. Let $X$ be a $G$-metric space and $f$ and $g$ be two self-mappings of $X$. Then $f$ and $g$ are called $R$-weakly commuting if there exists a positive real number $R$ such that $G(f g x, f g x, g f x) \leq R G(f x, f x, g x)$ holds for each $x \in X$. 
Two maps $f$ and $g$ are said to be compatible if, whenever $\left\{x_{n}\right\}$ in $X$ such that $\left\{f_{n}\right\}$ and $\left\{g x_{n}\right\}$ are G-convergent to some $t \in X$, then $\lim _{n \rightarrow \infty} G\left(f g x_{n}, f g x_{n}, g f x_{n}\right)=0$.

Example 1.8. Let $X=[0,2]$ with complete $G$-metric defined by

$$
G(x, y, z)=\max \{|x-y|,|x-z|,|y-z|\} .
$$

Let $f, g, S, T: X \rightarrow X$ defined by

$$
\begin{aligned}
& f x=1, x \geq 0, \\
& g x=\left\{\begin{array}{c}
1, x \in[0,1], \\
\frac{2-x}{2}, x \in(1,2],
\end{array},\right. \\
& S x=\left\{\begin{array}{c}
2-x, x \in[0,1], \\
x, x \in(1,2],
\end{array} \mid,\right.
\end{aligned}
$$

and

$$
T x=\left\{\begin{array}{c}
\frac{3-x}{2}, x \in[0,1] \\
\frac{x}{2}, x \in(1,2],
\end{array} \mid .\right.
$$

Then note that the pairs $\{f, S\}$ and $\{g, T\}$ are $R$-weakly commuting as they commute at their coincidence points. The pair $\{f, S\}$ is continuous compatible while the pair $\{g$, $T\}$ is non-compatible. To see that $g$ and $T$ are non-compatible, consider a decreasing sequence $\left\{x_{n}\right\}$ in $X$ such that $x_{n} \rightarrow 1$. Then $g x_{n} \rightarrow \frac{1}{2}, T x_{n} \rightarrow \frac{1}{2} \cdot g T x_{n}=\frac{4-x_{n}}{4} \rightarrow \frac{3}{4}$ and $\operatorname{Tg} x_{n}=\frac{2-x_{n}}{4} \rightarrow \frac{1}{4}$.

\section{Common fixed point theorems}

In this section, we obtain some unique common fixed point results for four mappings satisfying certain generalized contractive conditions in the framework of a generalized metric space. We start with the following result.

Theorem 2.1. Let $X$ be a complete G-metric space. Suppose that $\{f, S\}$ and $\{g, T\}$ be pointwise $R$-weakly commuting pairs of self-mappings on $X$ satisfying

$$
\begin{gathered}
G(f x, f x, g y) \leq h \max \{G(S x, S x, T y), G(f x, f x, S x), G(g y, g y, T y), \\
[G(f x, f x, T y)+G(g y, g y, S x)] / 2\}
\end{gathered}
$$

and

$$
\begin{gathered}
G(f x, g y, g y) \leq h \max \{G(S x, T y, T y), G(f x, S x, S x), G(g y, T y, T y), \\
[G(f x, T y, T y)+G(g y, S x, S x)] / 2\}
\end{gathered}
$$

for all $x, y \in X$, where $h \in[0,1)$. Suppose that $f X \subseteq T X, g X \subseteq S X$, and one of the pair $\{f, S\}$ or $\{g, T\}$ is compatible. If the mappings in the compatible pair are continuous, then $f, g, S$ and $T$ have a unique common fixed point.

Proof. Suppose that $f$ and $g$ satisfy the conditions (2.1) and (2.2). If $G$ is symmetric, then by adding these, we have

$$
\begin{aligned}
& d_{G}(f x, g y) \\
& \leq \frac{h}{2} \max \left\{d_{G}(S x, T y), d_{G}(f x, S x), d_{G}(g y, T y),\left[d_{G}(f x, T y)+d_{G}(g y, S x)\right] / 2\right\} \\
&+\frac{h}{2} \max \left\{d_{G}(S x, T y), d_{G}(f x, S x), d_{G}(g y, T y),\left[d_{G}(f x, T y)+d_{G}(g y, S x)\right] / 2\right\} \\
&= h \max \left\{d_{G}(S x, T y), d_{G}(f x, S x), d_{G}(g y, T y),\left[d_{G}(f x, T y)+d_{G}(g y, S x)\right] / 2\right\},
\end{aligned}
$$


for all $x, y \in X$ with $0 \leq h<1$, the existence and uniqueness of a common fixed point follows from [14]. However, if $X$ is non-symmetric $G$-metric space, then by the definition of metric $d_{G}$ on $X$ and (1.3), we obtain

$$
\begin{aligned}
& d_{G}(f x, g y) \\
&= G(f x, f x, g y)+G(f x, g y, g y) \\
& \leq \frac{2 h}{3} \max \left\{d_{G}(S x, T y), d_{G}(f x, S x), d_{G}(g y, T y),\left[d_{G}(f x, T y)+d_{G}(g y, S x)\right] / 2\right\} \\
&+\frac{2 h}{3} \max \left\{d_{G}(S x, T y), d_{G}(f x, S x), d_{G}(g y, T y),\left[d_{G}(f x, T y)+d_{G}(g y, S x)\right] / 2\right\} \\
&= \frac{4 h}{3} \max \left\{d_{G}(S x, T y), d_{G}(f x, S x), d_{G}(g y, T y),\left[d_{G}(f x, T y)+d_{G}\left(g y, S_{X}\right)\right] / 2\right\},
\end{aligned}
$$

for all $x, y \in X$. Here, the contractivity factor $\frac{4 h}{3}$ needs not be less than 1 . Therefore, metric $d_{G}$ gives no information. In this case, let $x_{0}$ be an arbitrary point in $X$. Choose $x_{1}$ and $x_{2}$ in $X$ such that $g x_{0}=S x_{1}$ and $f x_{1}=T x_{2}$. This can be done, since the ranges of $S$ and $T$ contain those of $g$ and $f$, respectively. Again choose $x_{3}$ and $x_{4}$ in $X$ such that $g x_{2}=S x 3$ and $f x_{3}=T x_{4}$. Continuing this process, having chosen $x_{n}$ in $X$ such that $g x_{2 n}$ $=S x_{2 n+1}$ and $f x_{2 n+1}=T x_{2 n+2}, n=0,1,2, \ldots$. Let

$$
y_{2 n}=S x_{2 n+1}=g x_{2 n} \text { and } y_{2 n+1}=T x_{2 n+2}=f x_{2 n+1} \text { for all } n=0,1,2, \ldots .
$$

For a given $n \in \mathbf{N}$, if $n$ is even, so $n=2 k$ for some $k \in \mathbf{N}$. Then from (2.1)

$$
\begin{aligned}
& G(\left.y_{n+1}, y_{n+1}, y_{n}\right) \\
&= G\left(y_{2 k+1}, y_{2 k+1}, y_{2 k}\right) \\
&= G\left(f x_{2 k+1}, f x_{2 k+1}, g x_{2 k}\right) \\
& \leq h \max \left\{G\left(S x_{2 k+1}, S x_{2 k+1}, T x_{2 k}\right), G\left(f x_{2 k+1}, f x_{2 k+1}, S x_{2 k+1}\right),\right. \\
&\left.G\left(g x_{2 k}, g x_{2 k}, T x_{2 k}\right),\left[G\left(f x_{2 k+1}, f x_{2 k+1}, T x_{2 k}\right)+G\left(g x_{2 k}, g x_{2 k}, S x_{2 k+1}\right)\right] / 2\right\} \\
&= h \max \left\{G\left(y_{2 k}, y_{2 k^{\prime}}, y_{2 k-1}\right), G\left(y_{2 k+1}, y_{2 k+1}, y_{2 k}\right),\right. \\
&\left.G\left(y_{2 k^{\prime}}, y_{2 k}, y_{2 k-1}\right),\left[G\left(y_{2 k+1}, y_{2 k+1}, y_{2 k-1}\right)+G\left(y_{2 k}, y_{2 k}, y_{2 k}\right)\right] / 2\right\} \\
& \leq h \max \left\{G\left(y_{2 k}, y_{2 k}, y_{2 k-1}\right), G\left(y_{2 k+1}, y_{2 k+1}, y_{2 k}\right),\right. \\
& {\left.\left[G\left(y_{2 k+1}, y_{2 k+1}, y_{2 k}\right)+G\left(y_{2 k^{\prime}}, y_{2 k}, y_{2 k-1}\right)\right] / 2\right\} } \\
&= h \max \left\{G\left(y_{n}, y_{n}, y_{n-1}\right), G\left(y_{n+1}, y_{n+1}, y_{n}\right)\right\} .
\end{aligned}
$$

This implies that

$$
G\left(y_{n+1}, y_{n+1}, y_{n}\right) \leq h G\left(y_{n}, y_{n}, y_{n-1}\right) .
$$

If $n$ is odd, then $n=2 k+1$ for some $k \in \mathbf{N}$. In this case (2.1) gives

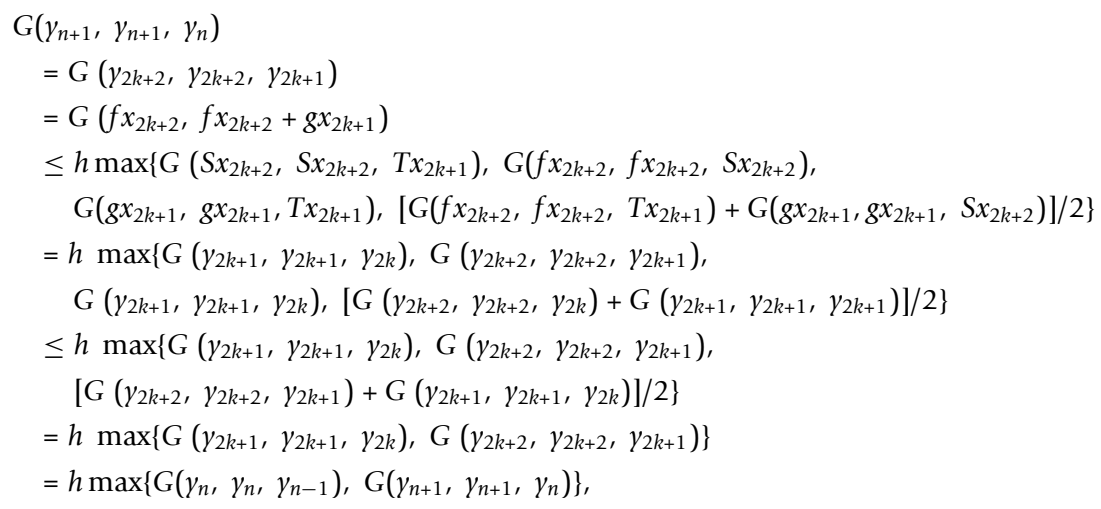


that is,

$$
G\left(y_{n+1}, y_{n+1}, y_{n}\right) \leq h G\left(y_{n}, y_{n}, y_{n-1}\right) .
$$

Continuing the above process, we have

$$
G\left(y_{n+1}, y_{n+1}, y_{n}\right) \leq h^{n} G\left(y_{1}, y_{1}, y_{0}\right) .
$$

Thus, if $y_{0}=y_{1}$, we get $G\left(y_{n}, y_{n+1}, y_{n+1}\right)=0$ for each $n \in \mathbf{N}$. Hence, $y_{n}=y_{n+1}$ for each $n \in \mathbf{N}$. Therefore, $\left\{y_{n}\right\}$ is G-Cauchy. So we may assume that $y_{0} \neq y_{1}$.

Let $n, m \in \mathbf{N}$ with $m>n$,

$$
\begin{aligned}
& G\left(y_{n}, y_{m}, y_{m}\right) \\
& \quad \leq G\left(y_{n}, y_{n+1}, y_{n+1}\right)+G\left(y_{n+1}, y_{n+2}, y_{n+2}\right)+\cdots+G\left(y_{m-1}, y_{m}, y_{m}\right) \\
& \quad \leq h^{n} G\left(y_{0}, y_{1}, y_{1}\right)+h^{n+1} G\left(y_{0}, y_{1}, y_{1}\right)+\cdots+h^{m-1} G\left(y_{0}, y_{1}, y_{1}\right) \\
& \quad=h^{n} G\left(y_{0}, y_{1}, y_{1}\right) \sum_{i=0}^{m-n-1} h^{i} \\
& \quad \leq \frac{h^{n}}{1-h} G\left(y_{0}, y_{1}, y_{1}\right),
\end{aligned}
$$

and so $G\left(y_{n}, y_{m}, y_{m}\right) \rightarrow 0$ as $m, n \rightarrow \infty$. Hence $\left\{y_{n}\right\}$ is a Cauchy sequence in $X$. Since $X$ is $G$-complete, there exists a point $z \in X$ such that $\lim _{n \rightarrow \infty} y_{n}=z$.

Consequently

$$
\lim _{n \rightarrow \infty} y_{2 n}=\lim _{n \rightarrow \infty} S x_{2 n+1}=\lim _{n \rightarrow \infty} g x_{2 n}=z
$$

and

$$
\lim _{n \rightarrow \infty} y_{2 n+1}=\lim _{n \rightarrow \infty} T x_{2 n+2}=\lim _{n \rightarrow \infty} f x_{2 n+1}=z .
$$

Let $f$ and $S$ be continuous compatible mappings. Compatibility of $f$ and $S$ implies that $\lim _{n \rightarrow \infty} G\left(f S x_{2 n+1}, f S x_{2 n+1}, S f x_{2 n+1}\right)=0$, that is $G(f z, f z, S z)=0$ which implies that $f z=$ $S z$. Since $f X \subset T X$, there exists some $u \in X$ such that $f z=T u$. Now from (2.1), we have

$G(f z, f z, g u) \leq h \max \{G(S z, S z, T u), G(f z, f z, S z), G(g u, g u, T u)$,

$[G(f z, f z, T u)+G(g u, g u, S z)] / 2\}$

$=h \max \{G(f z, f z, f z), G(f z, f z, f z), G(g u, g u, f z)$,

$[G(f z, f z, f z)+G(g u, g u, f z)] / 2\}$

$=h G(f z, g u, g u)$.

Also, from (2.2)

$$
\begin{aligned}
G(f z, g u, g u) \leq & h \max \{G(S z, T u, T u), G(f z, S z, S z), G(g u, T u, T u), \\
& {[G(f z, T u, T u)+G(g u, S z, S z)] / 2\} } \\
= & h \max \{G(f z, f z, f z), G(f z, f z, f z), G(g u, f z, f z), \\
& {[G(f z, f z, f z)+G(g u, f z, f z)] / 2\} } \\
= & h G(f z, f z, g u) .
\end{aligned}
$$

Combining above two inequalities, we get

$G(f z, f z, g u) \leq h^{2} G(f z, f z, g u)$. 
Since $h<1$, so that $f z=g u$. Hence, $f z=S z=g u=T u$. As the pair $\{g, T\}$ is $R$-weakly commuting, there exists $R>0$ such that

$$
G(g T u, g T u, T g u) \leq R G(g u, g u, T u)=0,
$$

that is, $g T u=T g u$. Moreover, $g g u=g T u=T g u=T T u$. Similarly, the pair $\{f, S\}$ is $R$ weakly commuting, there exists some $R>0$ such that

$$
G(f S z, f S z, S f z) \leq R G(f z, f z, S z)=0,
$$

so that $f S z=S f z$ and $f f z=f S z=S f z=S S z$.

Now by $(2.1)$

$$
\begin{aligned}
G(f f z, f f z, f z)= & G(f f z, f f z, g u) \\
\leq & h \max \{G(S f z, S f z, T u), G(f f z, f f z, S f z), G(g u, g u, T u), \\
& {[G(f f z, f f z, T u)+G(g u, g u, S f z)] / 2\} } \\
= & h \max \{G(f f z, f f z, g u), G(f f z, f f z, f f z), G(g u, g u, g u), \\
& {[G(f f z, f f z, g u)+G(g u, g u, f f z)] / 2\} } \\
= & h \max \{G(f f z, f f z, f z),[G(f f z, f f z, f z)+G(f z, f z, f f z)] / 2\} \\
= & \frac{h}{2}[G(f f z, f f z, f z)+G(f z, f z, f f z)],
\end{aligned}
$$

so that

$$
G(f f z, f f z, f z) \leq h G(f z, f z, f f z) .
$$

Again from (2.2), we have

$$
\begin{aligned}
G(f f z, f z, f z)= & G(f f z, g u, g u) \\
\leq & h \max \{G(S f z, T u, T u), G(f f z, S f z, S f z), G(g u, T u, T u), \\
& {\left.\left[G\left(f f_{z}, T u, T u\right)+G(g u, S f z, S f z)\right] / 2\right\} } \\
= & h \max \{G(S f z, g u, g u), G(f f z, f f z, f f z), G(g u, g u, g u), \\
& {[G(f f z, g u, g u)+G(g u, f f z, f f z)] / 2\} } \\
= & h \max \{G(f f z, f z, f z),[G(f f z, f z, f z)+G(f z, f f z, f f z)] / 2\} \\
= & \frac{h}{2}[G(f f z, f z, f z)+G(f f z, f f z, f z)],
\end{aligned}
$$

which implies

$$
G(f f z, f z, f z) \leq h G(f f z, f f z, f z) .
$$

From (2.5) and (2.6), we obtain

$$
G(f f z, f f z, f z) \leq h^{2} G(f f z, f f z, f z),
$$

and since $h^{2}<1$ so that $f f z=f z$. Hence, $f f z=S f z=f z$, and $f z$ is the common fixed point of $f$ and $S$. Since $g u=f z$, following arguments similar to those given above we conclude that $f z$ is a common fixed point of $g$ and $T$ as well. Now we show the uniqueness of fixed point. For this, assume that there exists another point $w$ in $X$ which is the common fixed point of $f, g, S$ and $T$. From (2.1), we obtain 


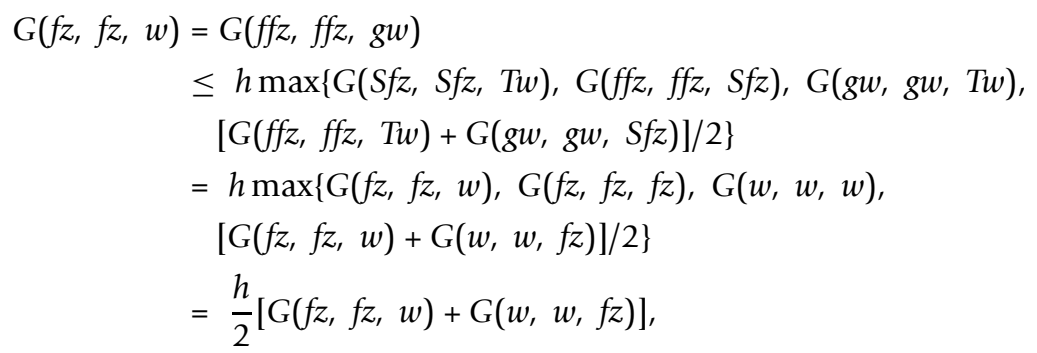

which implies that

$G(f z, f z, w) \leq h G(w, w, f z)$.

From (2.2), we get

$$
\begin{aligned}
G(f z, w, w)= & G(f f z, g w, g w) \\
\leq & h \max \{G(S f z, T w, T w), G(f f z, S f z, S f z), G(g w, T w, T w), \\
& {[G(f f z, T w, T w)+G(g w, S f z, S f z)] / 2\} } \\
= & h \max \{G(f z, w, w), G(f z, f z, f z), G(w, w, w), \\
& {[G(f z, w, w)+G(w, f z, f z)] / 2\} } \\
= & \frac{h}{2}[G(f z, w, w)+G(w, f z, f z)],
\end{aligned}
$$

which implies

$$
G(f z, w, w) \leq h G(f z, f z, w) .
$$

Now (2.7) and (2.8) give

$$
G(f z, f z, w) \leq h^{2} G(f z, f z, w),
$$

and $f z=w$. This completes the proof.

Example 2.2. Let $X=\{0,1,2\}$ with $G$-metric defined by

$$
(x, y, z) \quad G(x, y, z)
$$

$(0,0,0),(1,1,1),(2,2,2), \quad 0$

$(0,0,1),(0,1,0),(1,0,0)$,

$(0,0,2),(0,2,0),(2,0,0), \quad 1$

$(0,2,2),(2,0,2),(2,2,0)$,

$(0,1,1),(1,0,1),(1,1,0)$,

$(1,1,2),(1,2,1),(2,1,1)$,

$(1,2,2),(2,1,2),(2,2,1)$,

$(0,1,2),(0,2,1),(1,0,2), \quad 2$

$(1,2,0),(2,0,1),(2,1,0)$,

is a non-symmetric $G$-metric on $X$ because $G(0,0,1) \neq G(0,1,1)$.

Let $f, g, S, T: X \rightarrow X$ defined by

$\begin{array}{ccccc}x & f(x) & g(x) & S(x) & T(x) \\ 0 & 0 & 0 & 0 & 0 \\ 1 & 0 & 2 & 2 & 1 \\ 2 & 0 & 0 & 1 & 1\end{array}$

Then $f X \subseteq T X$ and $g X \subseteq S X$, with the pairs $\{f, S\}$ and $\{g$, $T\}$ are $R$-weakly commuting as they commute at their coincidence points. 
Now to get (2.1) and (2.2) satisfied, we have the following nine cases: (I) $x, y=0$, (II) $x=0, y=2$, (III) $x=1, y=0$, (IV) $x=1, y=2$, (V) $x=2, y=0$, (VI) $x=2, y=2$. For all these cases, $f(x)=g(y)=0$ implies $G(f x, f x, g y)=0$ and (2.1) and (2.2) hold.

(VII) For $x=0, y=1$, then $f x=0, g y=2, S x=0, T y=1$.

$G(f x, f x, g y)$

$=G(0,0,2)=1$

$\leq h \max \{1,0,2,1\}$

$=h \max \{G(0,0,1), G(0,0,0), G(2,2,1),[G(0,0,1)+G(2,2,0)] / 2\}$

$=h \max \{G(S x, S x, T y), G(f x, f x, S x), G(g y, g y, T y)$,

$[G(f x, f x, T y)+G(g y, g y, S x)] / 2\}$.

Thus, (2.1) is satisfied where $h=\frac{4}{5}$.

Also

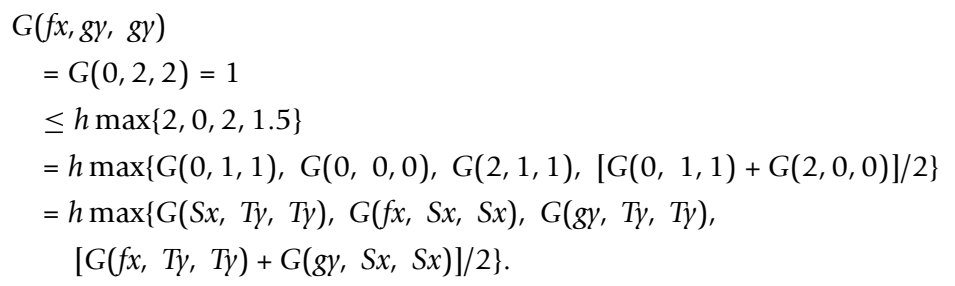

Thus, (2.2) is satisfied where $h=\frac{4}{5}$.

(VIII) Now when $x=1, y=1$, then $f x=0, g y=2, S x=2, T y=1$.

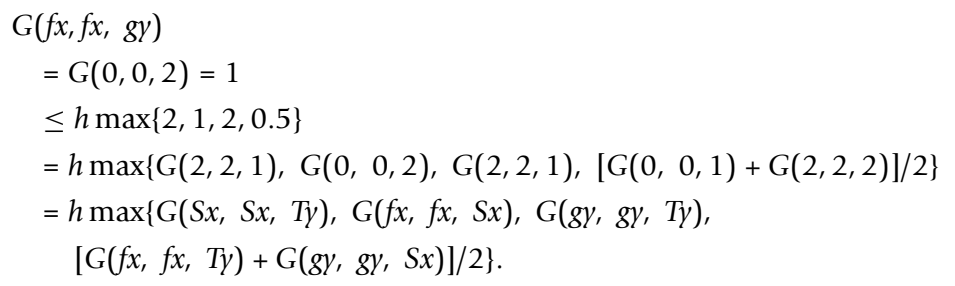

Thus, (2.1) is satisfied where $h=\frac{4}{5}$.

And

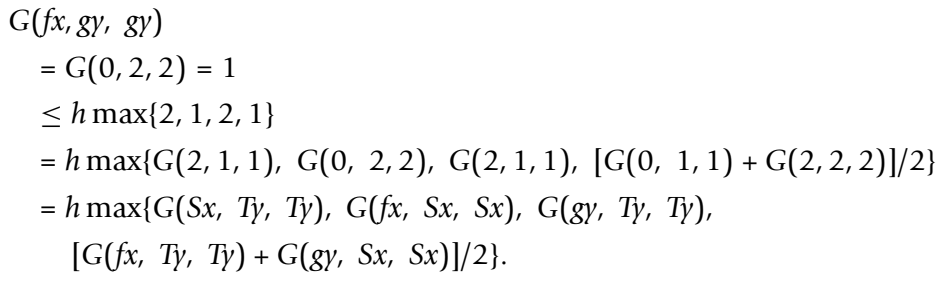

Thus, (2.2) is satisfied where $h=\frac{4}{5}$.

(IX) If $x=2, y=1$, then $f x=0, g y=2, S x=1, T y=1$ and

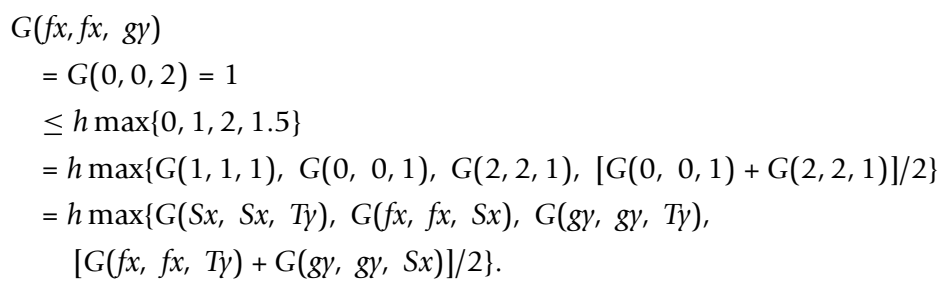


Thus, (2.1) is satisfied where $h=\frac{4}{5}$.

Also

$$
\begin{aligned}
& G(f x, g y, g y) \\
&= G(0,2,2)=1 \\
& \leq h \max \{0,2,2,2\} \\
&= h \max \{G(1,1,1), G(0,1,1), G(2,1,1),[G(0,1,1)+G(2,1,1)] / 2\} \\
&= h \max \{G(S x, T y, T y), G(f x, S x, S x), G(g y, T y, T y), \\
& {[G(f x, T y, T y)+G(g y, S x, S x)] / 2\} . }
\end{aligned}
$$

Thus, (2.2) is satisfied where $h=\frac{4}{5}$.

Hence, for all $x, y \in X,(2.1)$ and (2.2) are satisfied for $h=\frac{4}{5}<1$ so that all the conditions of Theorem 2.1 are satisfied. Moreover, 0 is the unique common fixed point for all of the mappings $f, g, S$ and $T$.

In Theorem 2.1, if we take $f=g$, then we have the following corollary.

Corollary 2.3. Let $X$ be a complete G-metric space. Suppose that $\{f, S\}$ and $\{f, T\}$ be pointwise $R$-weakly commuting pairs of self-mappings on $X$ satisfying

$$
\begin{gathered}
G(f x, f x, f y) \leq h \max \{G(S x, S x, T y), G(f x, f x, S x), G(f y, f y, T y), \\
[G(f x, f x, T y)+G(f y, f y, S x)] / 2\}
\end{gathered}
$$

and

$$
\begin{aligned}
G(f x, f y, f y) \leq & h \max \{G(S x, T y, T y), G(f x, S x, S x), G(f y, T y, T y)\} \\
& {[G(f x, T y, T y)+G(f y, S x, S x)] / 2\} }
\end{aligned}
$$

for all $x, y \in X$, where $h \in[0,1)$. Suppose that $f X \subseteq S X \cup T X$, and one of the pairs $\{f$, $S\}$ or $\{f, T\}$ is compatible. If the mappings in the compatible pair are continuous, then $f$, $S$ and $T$ have a unique common fixed point.

Also, if we take $S=T$ in Theorem 2.1, then we get the following.

Corollary 2.4. Let $X$ be a complete G-metric space. Suppose that $\{f, S\}$ and $\{g, S\}$ are pointwise $R$-weakly commuting pairs of self-maps on $X$ and

$$
\begin{aligned}
G(f x, f x, g y) \leq & h \max \{G(S x, S x, S y), G(f x, f x, S x), G(g y, g y, S y), \\
& {[G(f x, f x, S y)+G(g y, g y, S x)] / 2\} }
\end{aligned}
$$

and

$$
\begin{aligned}
G(f x, g y, g y) \leq & h \max \{G(S x, S y, S y), G(f x, S x, S x), G(g y, S y, S y), \\
& {[G(f x, S y, S y)+G(g y, S x, S x)] / 2\} }
\end{aligned}
$$

hold for all $x, y \in X$, where $h \in[0,1)$. Suppose that $f X \cup g X \subseteq S X$ and one of the pairs $\{f, S\}$ or $\{g, S\}$ is compatible. If the mappings in the compatible pair are continuous, then $f, g$ and $S$ have a unique common fixed point.

Corollary 2.5. Let $X$ be a complete $G$-metric space. Suppose that $f$ and $g$ are two self-mappings on $X$ satisfying

$$
\begin{gathered}
G(f x, f x, g y) \leq h \max \{G(x, x, y), G(f x, f x, x), G(g y, g y, y), \\
[G(f x, f x, y)+G(g y, g y, x)] / 2\}
\end{gathered}
$$


and

$$
\begin{aligned}
G(f x, g y, g y) \leq & h \max \{G(x, y, y), G(f x, x, x), G(g y, y, y), \\
& {[G(f x, y, y)+G(g y, x, x)] / 2\} }
\end{aligned}
$$

for all $x, y \in X$, where $h \in[0,1)$. Suppose that one of $f$ or $g$ is continuous, then $f$ and $g$ have a unique common fixed point.

Proof. Taking $S$ and $T$ as identity maps on $X$, the result follows from Theorem 2.1.

Corollary 2.6. Let $X$ be a complete $G$-metric space and $f$ be a self-map on $X$ such that

$G(f x, f x, f y) \leq h \max \{G(x, x, y), G(f x, f x, x), G(f y, f y, y)$,

$$
[G(f x, f x, y)+G(f y, f y, x)] / 2\}
$$

and

$G(f x, f y, f y) \leq h \max \{G(x, y, y), G(f x, x, x), G(f y, y, y)$,

$$
[G(f x, y, y)+G(f y, x, x)] / 2\}
$$

hold for all $x, y \in X$, where $h \in[0,1)$. Then $f$ has a unique fixed point.

Proof. If we take $f=g$, and $S$ and $T$ as identity maps on $X$, then from $f$ has a unique fixed point by Theorem 2.1.

\section{Application}

Let $\Omega=[0,1]$ be bounded open set in $\mathbb{R}, L^{2}(\Omega)$, the set of functions on $\Omega$ whose square is integrable on $\Omega$. Consider an integral equation

$$
p(t, x(t))=\int_{\Omega} q(t, s, x(s)) d s
$$

where $p: \Omega \times \mathbb{R} \rightarrow \mathbb{R}$ and $q: \Omega \times \Omega \times \mathbb{R} \rightarrow \mathbb{R}$ be two mappings. Define $G: X \times X \times$ $X \rightarrow \mathbb{R}_{+}$by

$$
G(x, y, z)=\sup _{t \in \Omega}|x(t)-y(t)|+\sup _{t \in \Omega}|y(t)-z(t)|+\sup _{t \in \Omega}|z(t)-x(t)| .
$$

Then $X$ is a G-complete metric space. We assume the following that is there exists a function $G: \Omega \times \mathbb{R} \rightarrow \mathbb{R}^{+}$:

(i) $p(s, v(t)) \geq \int_{\Omega} q(t, s, u(s)) d s \geq G(s, v(t))$ for each $s, t \in \Omega$..

(ii) $p(s, v(t))-G(s, v(t)) \leq h|p(s, v(t))-v(t)|$.

Then integral equation (3.1) has a solution in $L^{2}(\Omega)$.

Proof. Define $(f x)(t)=p(t, x(t))$ and $(g x)(t)=\int_{\Omega} q(t, s, x(s)) d s$. Now

$$
\begin{aligned}
G(f x, f x, g y) & =2 \sup _{t \in \Omega}|(f x)(t)-(g y)(t)| \\
& =2 \sup _{t \in \Omega}\left|p(t, x(t))-\int_{\Omega} q(t, s, y(t)) d t\right| \\
& \leq 2 \sup _{t \in \Omega}|p(t, x(t))-G(t, x(t))| \\
& \leq 2 h \sup _{t \in \Omega}|p(t, x(t))-x(t)| \\
& =h G(f x, f x, x) .
\end{aligned}
$$


Thus

$$
\begin{gathered}
G(f x, f x, g y) \leq h \max \{G(x, x, y), G(f x, f x, x), G(g y, g y, y), \\
[G(f x, f x, y)+G(g y, g y, x)] / 2\}
\end{gathered}
$$

is satisfied. Similarly (2.14) is satisfied. Now we can apply Corollary 2.5 to obtain the solution of integral equation (3.1) in $L^{2}(\Omega)$.

Remark 1. Theorems 2.8-2.9 in [3] and Corollaries 2.6-2.8 in [4] are special cases of our results Theorem 2.1 and Corollaries 2.3-2.6.

Remark 2. A $G$-metric naturally induces a metric $d_{G}$ given by $d_{G}(x, y)=G(x, y, y)+$ $G(x, x, y)$. If the $G$-metric is not symmetric, the inequalities (2.1) and (2.2) do not reduce to any metric inequality with the metric $d_{G}$. Hence, our theorems do not reduce to fixed point problems in the corresponding metric space $\left(X, d_{G}\right)$.

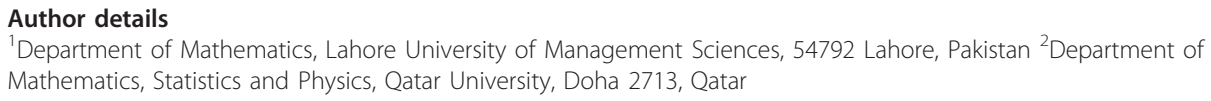

1. Mustafa, Z, Sims, B: Some remarks concerning D-metric spaces. Proceedings of the International Conference on Fixed Point Theory and Applications, Valencia, Spain. 189-198 (2003)

2. Mustafa, Z, Sims, B: A new approach to generalized metric spaces. J Nonlinear Convex Anal. 7(2), 289-297 (2006)

3. Mustafa, Z, Obiedat, H, Awawdeh, F: Some fixed point theorem for mapping on complete G-metric spaces. Fixed Point Theor Appl 2008, 12 (2008). Article ID 189870

4. Mustafa, Z, Sims, B: Fixed point theorems for contractive mapping in complete G-metric spaces. Fixed Point Theor Appl 2009, 10 (2009). >Article ID 917175

5. Mustafa, Z, Shatanawi, W, Bataineh, M: Existence of fixed point results in G-metric spaces. Int J Math Math Sci 2009,10 (2009). Article ID 283028

6. Mustafa, Z, Awawdeh, F, Shatanawi, W: Fixed point theorem for expansive mappings in G-metric spaces. Int J Contemp Math Sci. 5, 2463-2472 (2010)

7. Abbas, M, Rhoades, BE: Common fixed point results for non-commuting mappings without continuity in generalized metric spaces. Appl Math Comput. 215, 262-269 (2009). doi:10.1016/j.amc.2009.04.085

8. Abbas, M, Nazir, T, Radenović, S: Some periodic point results in generalized metric spaces. Appl Math Comput. 217, 4094-4099 (2010). doi:10.1016/j.amc.2010.10.026

9. Chugh, R, Kadian, T, Rani, A, Rhoades, BE: Property $p$ in G-metric spaces. Fixed Point Theor Appl 2010, 12 (2010). Article ID 401684

10. Saadati, R, Vaezpour, SM, Vetro, P, Rhoades, BE: Fixed point theorems in generalized partially ordered G-metric spaces. Math Comput Modelling. 52(5-6), 797-801 (2010). doi:10.1016/j.mcm.2010.05.009

11. Shatanawi, W: Fixed point theory for contractive mappings satisfying $\Phi$-maps in G-metric spaces. Fixed Point Theor Appl 2010, 9 (2010). Article ID 181650

12. Abbas, M, Khan, AR, Nazir, T: Coupled common fixed point results in two generalized metric spaces. Appl Math Comput. 217, 6328-6336 (2011). doi:10.1016/j.amc.2011.01.006

13. Choudhury, BS, Maity, P: Coupled fixed point results in generalized metric spaces. Math Comput Modelling. 54, 73-79 (2011). doi:10.1016/j.mcm.2011.01.036

14. Pant, RP: R-weak commutativity and common fixed points. Soochow J Math. 1(25), 37-42 (1999)

doi:10.1186/1687-1812-2011-41

Cite this article as: Abbas et al:: Common fixed points of $R$-weakly commuting maps in generalized metric spaces. Fixed Point Theory and Applications 2011 2011:41. 